\title{
1 JACKIE: Fast enumeration of single- and multi-copy CRISPR binding sites
}

2 Jacqueline Jufen Zhu $^{1}$, Albert Wu Cheng ${ }^{1,2,3,4,5}$

3 1. The Jackson Laboratory for Genomic Medicine, Farmington, CT 06032, USA

4 2. The Jackson Laboratory Cancer Center, Bar Harbor, ME 04609, USA

5 3. Department of Genetics and Genome Sciences, University of Connecticut Health

6 Center, Farmington, CT 06030, USA

74 Institute for Systems Genomics, University of Connecticut Health Center, Farmington,

8 CT 06030, USA

$9 \quad 5 . \quad$ Correspondence: albert.cheng@jax.org

\section{ABSTRACT}

12 Summary: CRISPR-based methods for genome, epigenome editing and imaging have provided

13 powerful tools to interrogate the functions of the genome. The design of guide RNA (gRNA) is a

14 vital step of CRISPR experiments. We report here the implementation of JACKIE (Jackie and

15 Albert's CRISPR K-mer Instances Enumerator), a pipeline for enumerating all potential single-

16 and multi-copy CRISPR sites in the genome. We demonstrate the application of JACKIE to

17 identify locus-specific repetitive sequences for CRISPR/Casilio-based genomic labeling.

19 Availability: Source codes and CRISPR site databases (JACKIEdb) for hg38 and mm10 are

20 available for download at http://crispr.software/JACKIE

\section{1. INTRODUCTION}

23 CRISPR/Cas technologies have revolutionized genome research by providing versatile tools for

24 "writing" the genome, epigenome and transcriptome as well as "reading" the dynamics of the

25 genome through live cell genomic imaging techniques (Sander and Joung, 2014). Genomic 
26 imaging by CRISPR/Cas-based technologies require clustered repetitive sequences (Cheng, et

27 al., 2016; Ma, et al., 2018; Maass, et al., 2018; Qin, et al., 2017). dCas9-based gene activation

28 and epigenome editing also benefited from synergistic binding events (Cheng, et al., 2016;

29 Cheng, et al., 2013; Maeder, et al., 2013; Mali, et al., 2013; Taghbalout, et al., 2019). CRISPR-

30 mediated gene deletion may be induced using a pair of gRNAs flanking the region of interest

31 (Canver, et al., 2017) while other CRISPR applications may benefit from a database of unique

32 CRISPR binding sites.

33 For fast enumeration of all potential CRISPR binding sites and their locations of

34 occurrences in the genome, we have implemented JACKIE (Jackie and Albert's CRISPR K-mer

35 Instances Enumerator) pipeline that is compatible with high performance computing (HPC)

36 clusters. We further provide helper scripts for extracting binding sites within regions of interest,

37 as well as a wrapper script to run Cas-OFFinder (Bae, et al., 2014) to identify off-target profiles

38 of selected CRISPR binding sites.

\section{IMPLEMENTATION}

41 JACKIE first scans the genome and enumerates any potential SpCas9 binding sites with the

42 PAM constraint ( $\mathrm{nGG}$ ). To allow memory and computation efficiency, sequences are encoded

43 into an unsigned 64-bit integer (NucKey) by encoding each nucleotide in 3 bits (Fig 1). The

44 chromosome and location for each instance are also encoded as integer values. The NucKey,

45 chromosome and location (KeyedPosition) are written into files prefixed by a predefined prefix

46 length (default to 6). The second step: Each bin file is loaded into a list in memory, and sorted

47 by NucKey, thus placing records of CRISPR sites with identical sequences adjacent to each

48 other in the list. The sequence-sorted list is then traversed to output CRISPR sites in bed

49 interval files. The extensive divide-and-conquer design allows JACKIE to be highly parallelized

50 on an HPC cluster (Fig 1). 
Helper scripts were written to take the bed file outputs from JACKIE to further collapse

52 same-chromosome CRISPR binding sites into an extended bed interval file format with each

53 record containing the CRISPR binding sites encoded as blocks. Such extended bed files can

54 then be loaded onto genome browsers for visualization. Other helper scripts were written for

55 downstream processing, such as for the fast extraction of CRISPR sites overlapping defined set

56 of intervals and automated queries into an offline version of Cas-OFFinder (Bae, et al., 2014) to

57 compute off-target profiles for selected sgRNA sequences. sgRNA sequences, number of

58 occurrences, and span sizes (the distance between the 5' most site to 3' most site for CRISPR

59 sites on the same chromosome) are encoded in the output files so that CRISPR sites can be

60 filtered by simple text processing software (e.g., awk) to identify sites for specific purposes. We

61 used JACKIE to generate CRISPR binding site databases (JACKIEdb) for human genome

62 version hg38 and mouse genome $\mathrm{mm} 10$, which took $\sim 35 \mathrm{~min}$ and $\sim 22 \mathrm{~min}$, respectively, on an

63 HPC cluster with 2,568 Intel Xeon compute cores and >38TB of system memory.

64

\section{USE CASES}

66 Some potential applications of JACKIE pipeline are illustrated in Fig 2. For example, highly 67 repetitive sequences (>30 copies) and low repetitive arrays ( $\sim 6$ copies) can be used for imaging 68 applications (Fig 2a) (Cheng, et al., 2016; Ma, et al., 2018; Maass, et al., 2018; Qin, et al., 2017).

69 These sites can be identified from JACKIE output by filtering for the desired range of repeat 70 number and span size. To demonstrate such use case, we have selected three CRISPR binding 71 sequences with $>30$ copies clustered specifically within $10 \mathrm{~kb}$ region. We then applied Casilio

72 imaging to visualize the sequence clusters via fluorescence microscopy (Fig $2 \mathrm{~b}$ ), producing 3

73 fluorescence foci in each case, consistent with the ploidy of the HEK293T cells used in the

74 experiments. Similarly, promoter-specific repeats might be identified (e.g., >3 copies, within $1 \mathrm{~kb})$

75 overlapping gene promoters for synergistic gene activation or repression by CRISPRa or

76 CRISPRi technologies (Fig 2c) (Cheng, et al., 2016; Cheng, et al., 2013; Maeder, et al., 2013; 
77 Mali, et al., 2013; Taghbalout, et al., 2019). Two or more copy sites flanking or spanning a

78 region or interest might be identified for inducing deletion or complex chromosome

79 rearrangements (Fig 2d). (Canver, et al., 2017; Choi and Meyerson, 2014; Maddalo, et al., 2014;

80 Maresch, et al., 2016).

81 Although currently designed for SpCas9 binding site enumeration, JACKIE can be

82 modified to identify binding sites for other CRISPR/Cas systems by changing the code segment

83 written for the PAM requirement filtering. Alternatively, PAM requirement might be removed from

84 the codes to enable identification of all k-mer sites and their occurrences in the genome. The

85 use of one uint64 for encoding NucKey limits the enumeration of sequences up to 20mer. By

86 using multiple NucKey, or a variable-size vector of NucKey, longer k-mer sequences can be

87 enumerated.

88

89 Experimental Material \& Methods

90 sgRNA spacer sequences (as listed in Fig 2b) were cloned into sgRNA-15xPBSc expression

91 vector via an oligo-annealing protocol as previously described (Cheng, et al., 2016). HEK293T

92 cells were cultivated in Dulbecco's modified Eagle's medium (DMEM) (Sigma) with 10\% fetal

93 bovine serum (FBS)(Lonza), 4\% Glutamax (Gibco), 1\% Sodium Pyruvate (Gibco) and penicillin-

94 streptomycin (Gibco). Incubator conditions were $37^{\circ} \mathrm{C}$ and $5 \% \mathrm{CO}$. Cells were seeded into 24-

95 well plates the day before being transfected with 50ng pAC1445-dCas9 plasmid (Addgene

96 \#73169), 25ng pAC1447-Clover-PUFa plasmid (Addgene \#73689) and 250ng sgRNA-15xPBSc

97 plasmid. Cells were fixed, mounted on slides, and imaged 48 hours after transfection on a Leica

98 SP8 confocal microscope.

99 


\section{Acknowledgement}

101 This work has been supported by the Jackson Laboratory internal grants (to A.C.) and National

102 Human Genome Research Institute 1R01HG009900 (to A.C).

\section{Figure Legends}

105 Fig 1. Implementation of JACKIE. (a) JACKIE first scans the genome for potential SpCas9

106 binding sites, and encode each occurrence in a data structure called KeyedPosition, consisting

107 of NucKey (uint64), chrID (uint32), and position (int32). NucKey records a binary representation

108 of the 20-mer spacer sequence using 3 bits per nucleotide. chrlD records an integer

109 representing a chromosome. A chrlD-chromosome mapping file is generated automatically.

110 Position records the location of the binding site, with negative and positive integers representing

111 the minus strand and positive strand, respectively. JACKIE outputs KeyedPosition as it scans

112 the genome. For efficient sorting in the next step, KeyedPosition records are appended to a file

113 named by the 6-mer prefix (<6merPrefix>.bin). To parallelize this step, four processes are

114 started as separate cluster jobs, each focusing on the A, C, T, or G as the first nucleotide of

115 binding sites. (b) In the second step, JACKIE loads each <6merPrefix > bin file and performs a

116 sort on the KeyedPosition records on the NucKey variable using std_sort routine and then

117 traverse the sorted list of KeyedPosition to output a bed interval file containing the coordinate

118 position of each CRISPR sites with item name encoded by

$119<$ NucKey>.<copyNumber>/<sequence>, and score field recording the copy number.

120 Parallelization is achieved by either starting separate job on individual $<6$ merPrefix $>$.bin files, or

121 by starting batch jobs focusing on 2 mer prefices (e.g., AA AT AC AG operating on AA*.bin,

$122 A T^{*}$.bin, $A C^{*}$.bin, $A G^{*}$.bin, etc). The outputs (<6merPrefix $>$.bed) are then merged into a

123 combined bed file using cat unix command. Helper scripts are provided for downstream

124 processing of the bed file into collapsed bed file with each record encoding all binding sites of 
125 the same sequence in the same chromosome, for extracting binding sites within defined

126 regions, or for running the Cas-OFFinder program to identify off-target profiles of selected

127 sequences.

128 Fig 2. Potential applications of JACKIE pipeline. (a) JACKIE can be used to identify

129 clustered CRISPR binding sites with high copy number or low copy number for CRISPR-based

130 genomic imaging experiments. (b) To demonstate genomic imaging use case, we filtered

131 sgRNA sequences with $>30$ copies clustered within specific $<10 \mathrm{~kb}$ regions and performed

132 Casilio imaging experiment using designed sgRNAs. UCSC genome browser screenshots are

133 shown on the left with the line-bar annotating the CRISPR sites, and fluorescence microscopy

134 images shown on the right. Microscopy images were derived by merging green (Casilio-

135 labeling) and blue (DAPI: nucleus stain). Arrows point to fluorescent loci of interest. Scale bars

136 indicate $5 \mu \mathrm{m}$. (c) JACKIE can be used to identify clustered CRISPR binding sites for synergistic

137 activation or repression of target cis-regulatory elements (e.g., promoters) using CRISPR-based

138 epigenetic editing approaches (e.g., CRISPRa or CRISPRi). (d) JACKIE can identify unique

139 CRISPR binding sites in the genome for precise indel induction, dual CRISPR sites for targeted

140 deletion, or clustered sites spanning a genomic region to induce complex rearrangement

141 events. 
a
chr11
1532633
PAM
ATGATTCTAGGTCAGTCCGTAGG
seq=“ATGATTCTAGGTCAGTCCGT”

ATGATT.bin

101010101010101010100101

1010101010101101010101001001
1110100101010101010100010101

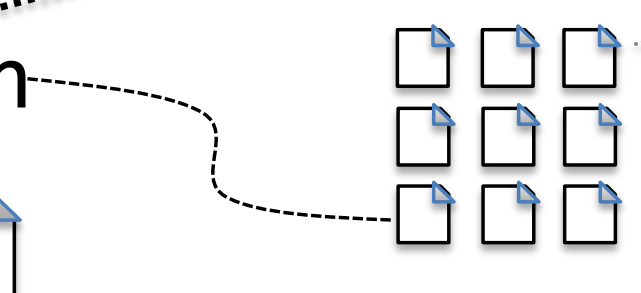

\section{KeyedPosition}

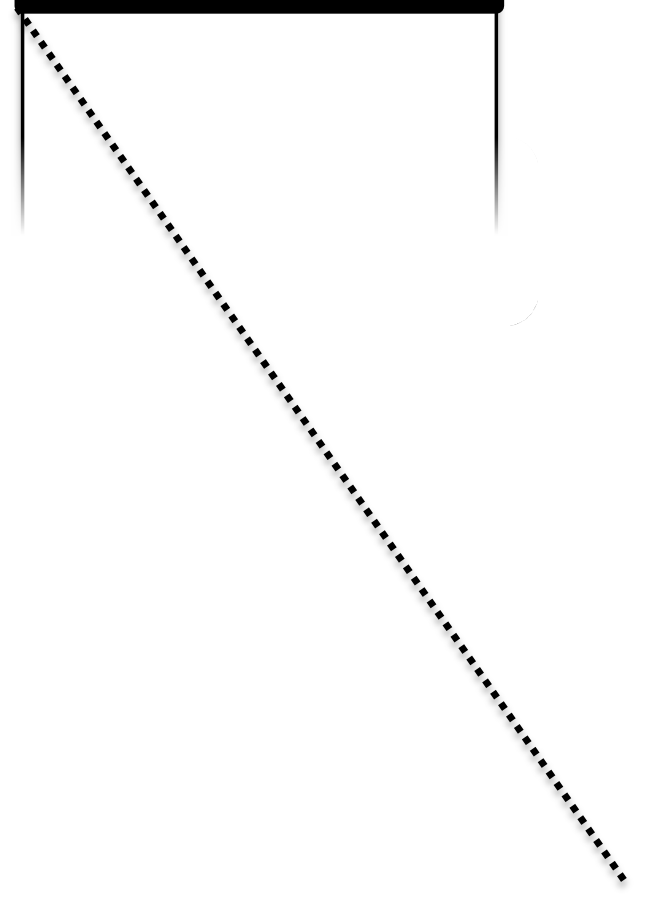

KeyedPosition

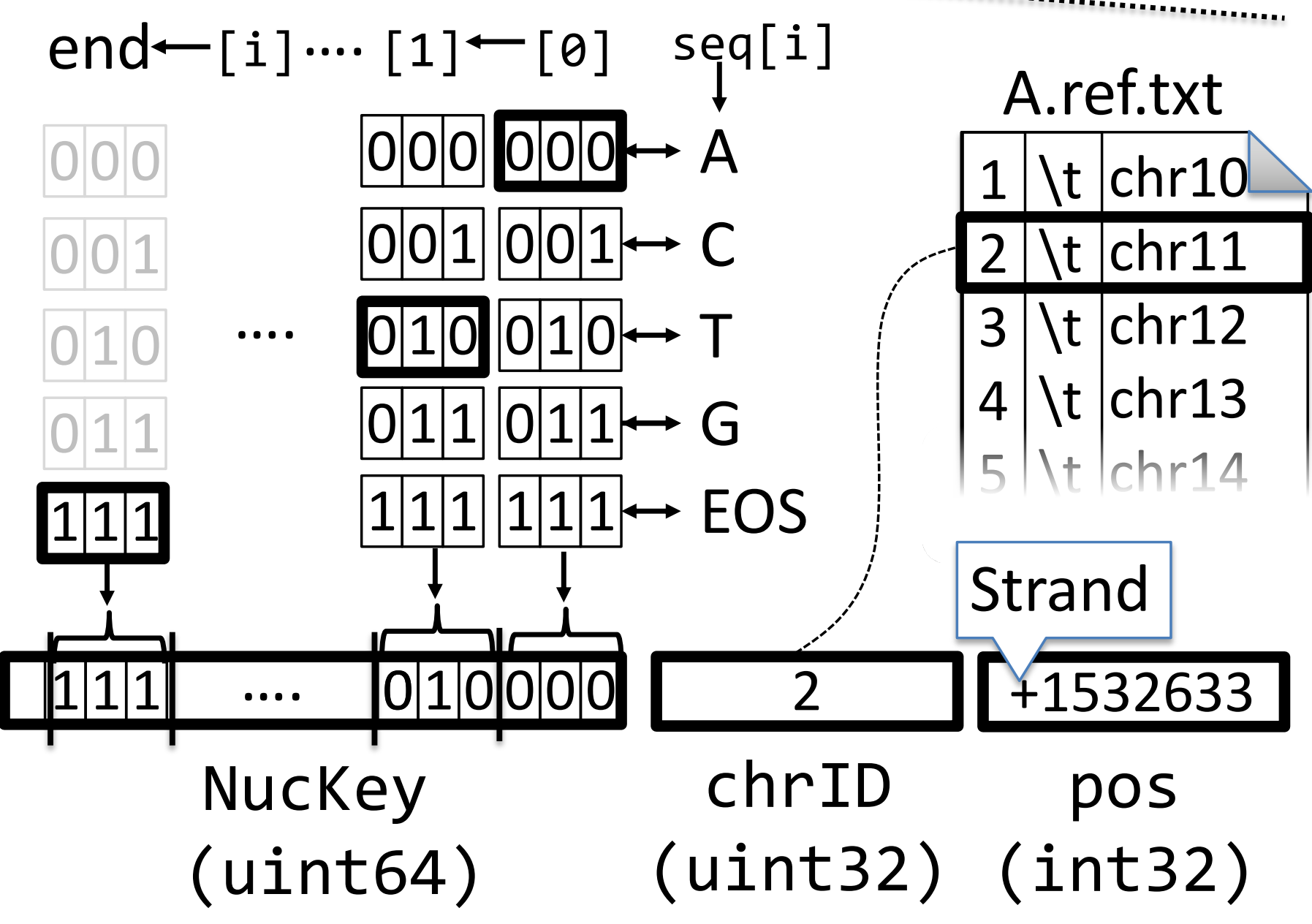

b

ATGATT . bin
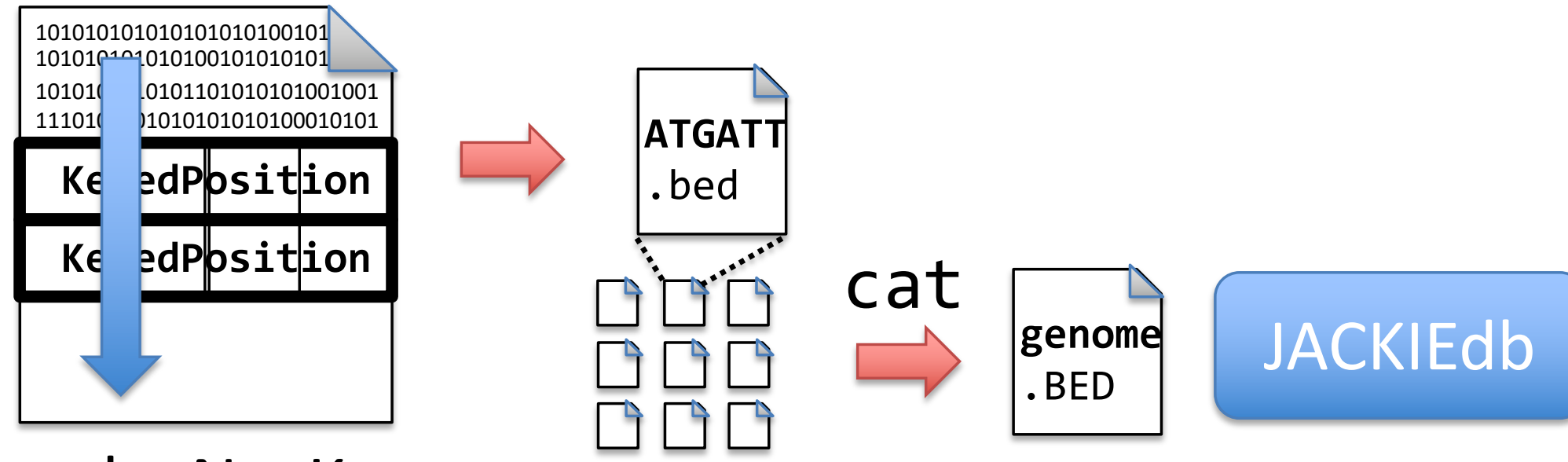

Sort by NucKey 
a. High- and low-repeat imaging

Copy number $>30$ (high) or $\sim 6$ (low)

Span size $<10 \mathrm{~kb}$

GFP

Figure 2

JACKIEdb

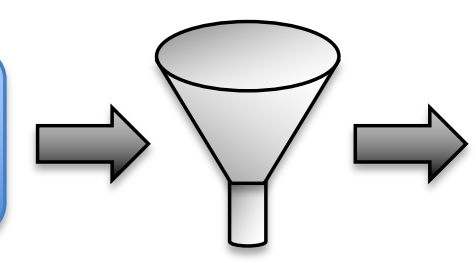

$\Omega$ dCas9 9
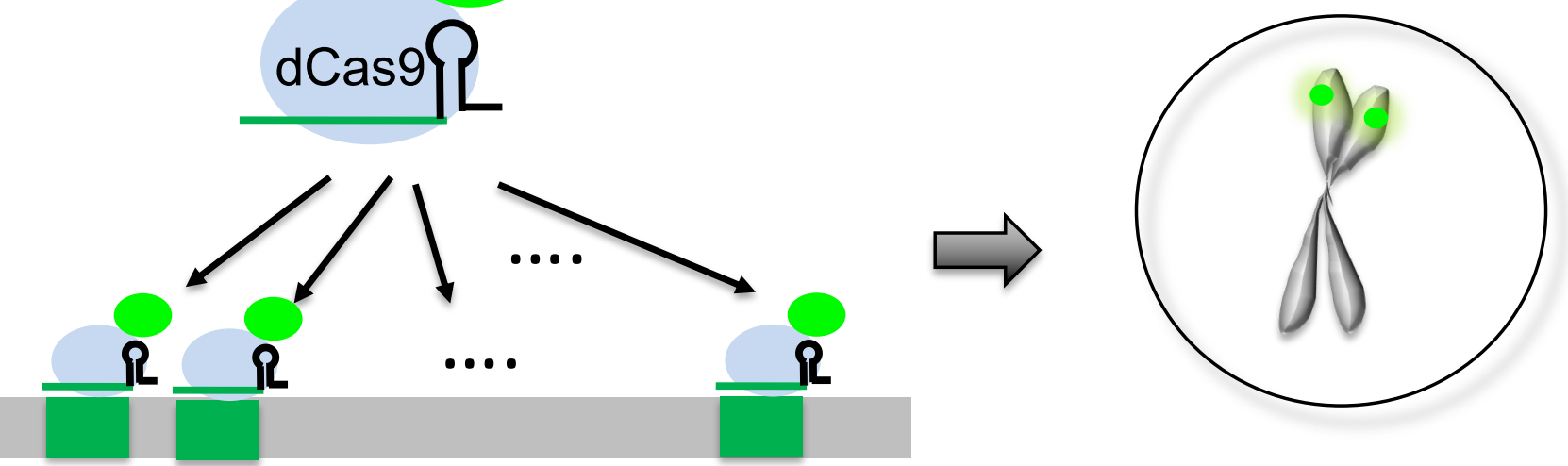

b. Examples sgRNA design for imaging (hg38, >30 copies, <10kb)

i. GGTGTAGACAGTGGAGCAGC, chr10:1046518-1047610, 33 copies

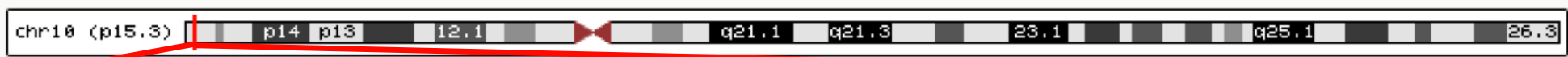

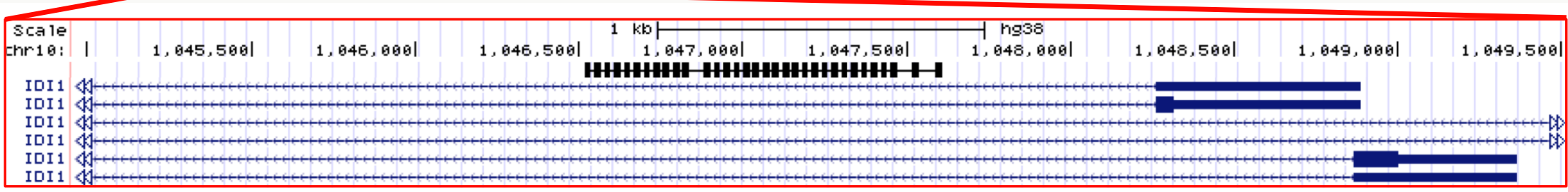

ii. TGAGGACTGAATCTAGAACC, chr18:79033234-79037027, 52 copies

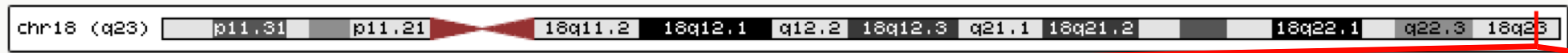

\begin{tabular}{|c|c|c|c|c|c|c|}
\hline \multirow[t]{2}{*}{$\begin{array}{c}\text { scale } \\
\text { chris: }\end{array}$} & I & $79,632,0901$ & $79,055,0001$ & H, & $79,058,0001$ & $79,059,0001$ \\
\hline & & & & No gene features & & \\
\hline
\end{tabular}

iii. ACTAACACTGCAGCGACCCC, chr4 744703-747683, 53 copies

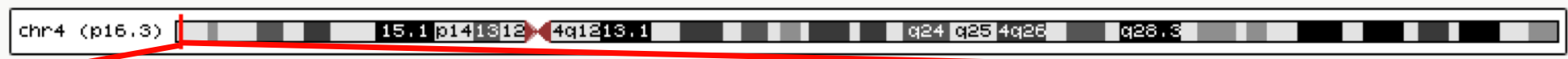

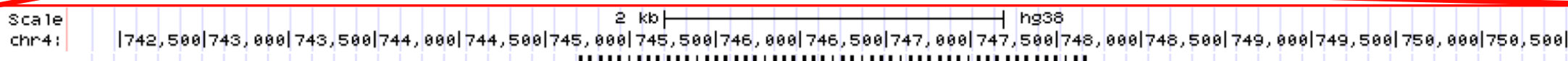
FCGF3 11 -

-

\section{c. Synergistic CRISPRa/i}

\section{VP64}

Copy number $>=3$

Span size $<1 \mathrm{~kb}$

Overlap promoter

\section{JACKIEdb}
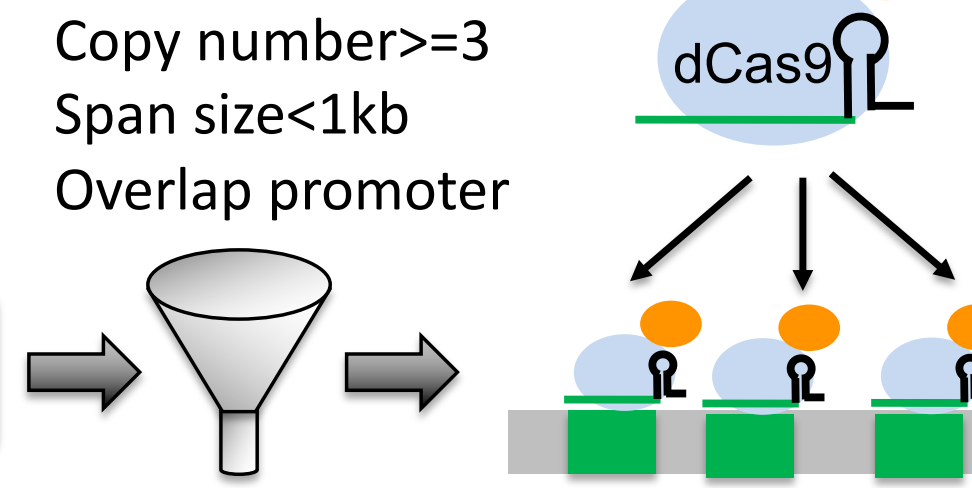

$\Omega \Omega \Omega$
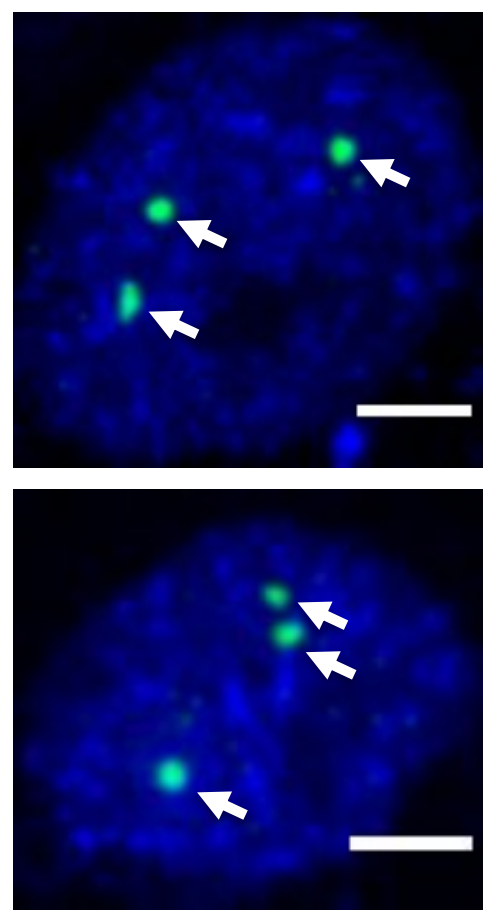

d. Genome editing applications

Copy number $=1$

Cas9 \{

indels

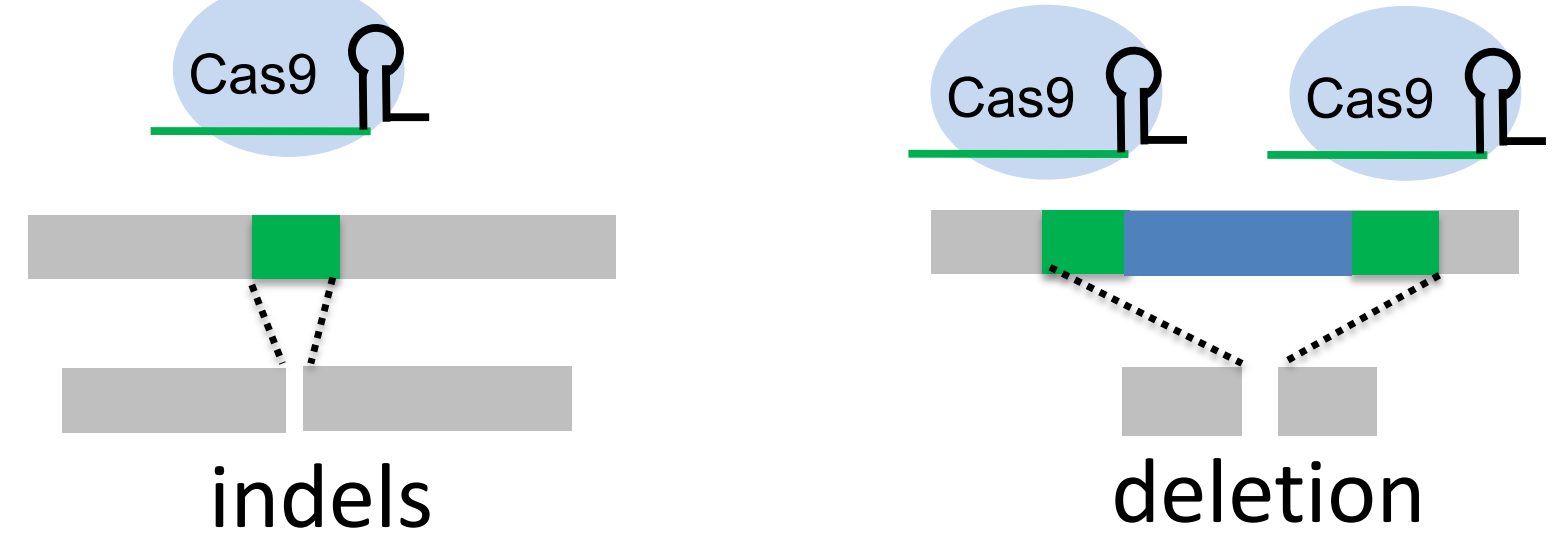

Copy number $=2$

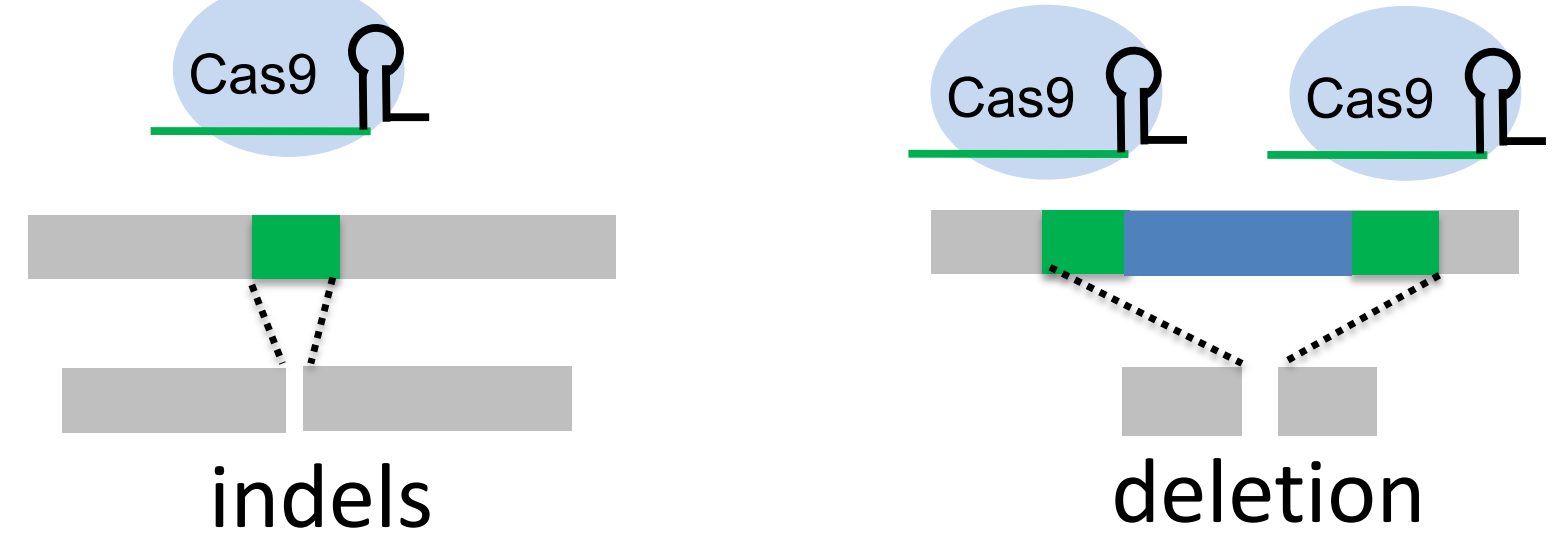

Copy number $>2$

Gene X
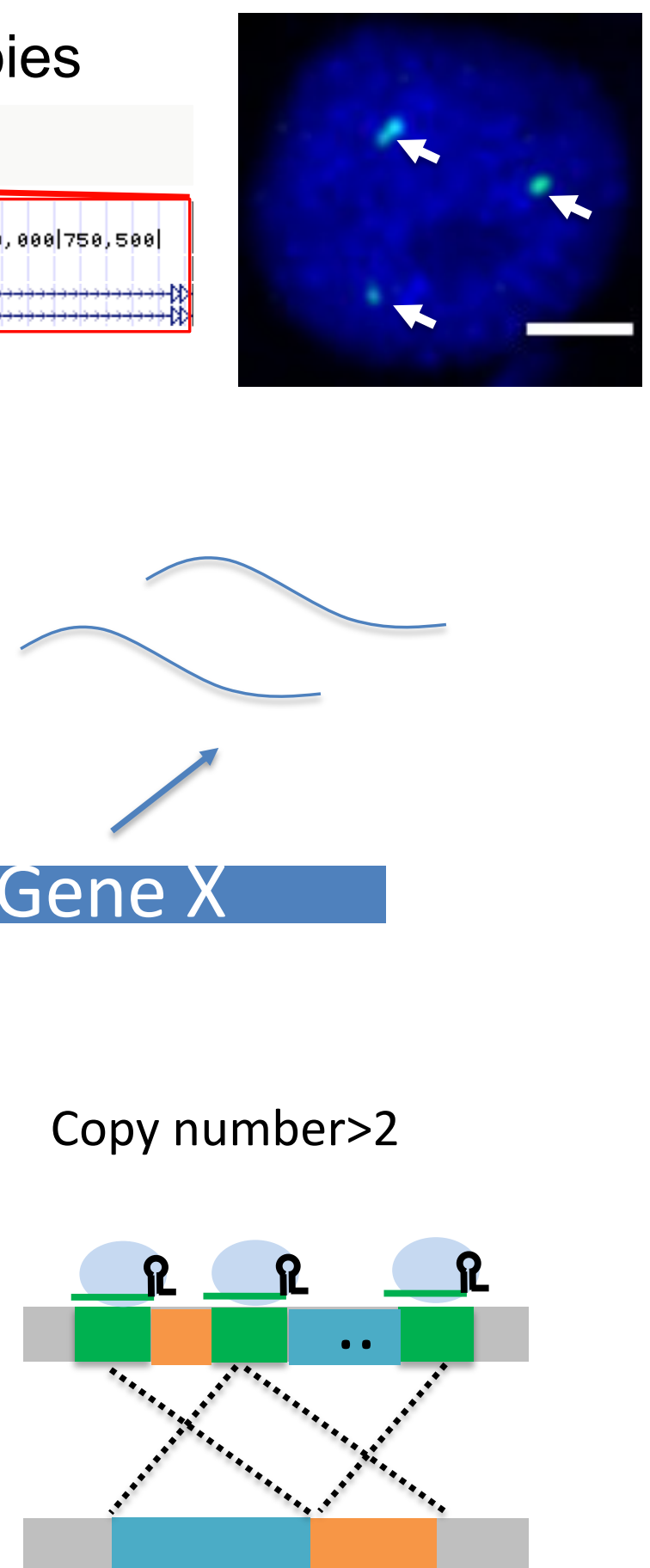

Rearrangements 


\section{References}

143 Bae, S., Park, J. and Kim, J.S. Cas-OFFinder: a fast and versatile algorithm that searches for

144 potential off-target sites of Cas9 RNA-guided endonucleases. Bioinformatics (Oxford, England)

145 2014;30(10):1473-1475.

146 Canver, M.C., et al. Characterization of genomic deletion efficiency mediated by clustered

147 regularly interspaced short palindromic repeats (CRISPR)/Cas9 nuclease system in mammalian

148 cells. The Journal of biological chemistry 2017;292(6):2556.

149 Cheng, A.W., et al. Casilio: a versatile CRISPR-Cas9-Pumilio hybrid for gene regulation and

150 genomic labeling. Cell research 2016;26(2):254-257.

151 Cheng, A.W., et al. Multiplexed activation of endogenous genes by CRISPR-on, an RNA-guided

152 transcriptional activator system. Cell research 2013;23(10):1163-1171.

153 Choi, P.S. and Meyerson, M. Targeted genomic rearrangements using CRISPR/Cas

154 technology. Nature communications 2014;5:3728.

$155 \mathrm{Ma}, \mathrm{H}$., et al. CRISPR-Sirius: RNA scaffolds for signal amplification in genome imaging. Nature

156 methods 2018;15(11):928-931.

157 Maass, P.G., et al. Spatiotemporal allele organization by allele-specific CRISPR live-cell

158 imaging (SNP-CLING). Nature structural \& molecular biology 2018;25(2):176-184

159 Maddalo, D., et al. In vivo engineering of oncogenic chromosomal rearrangements with the

160 CRISPR/Cas9 system. Nature 2014;516(7531):423-427.

161 Maeder, M.L., et al. CRISPR RNA-guided activation of endogenous human genes. Nature

162 methods 2013;10(10):977-979.

163 Mali, P., et al. CAS9 transcriptional activators for target specificity screening and paired

164 nickases for cooperative genome engineering. Nature biotechnology 2013;31(9):833-838.

165 Maresch, R., et al. Multiplexed pancreatic genome engineering and cancer induction by

166 transfection-based CRISPR/Cas9 delivery in mice. Nature communications 2016;7:10770. 
167 Qin, P., et al. Live cell imaging of low- and non-repetitive chromosome loci using CRISPR-Cas9.

168 Nature communications 2017;8:14725.

169 Sander, J.D. and Joung, J.K. CRISPR-Cas systems for editing, regulating and targeting

170 genomes. Nature biotechnology 2014;32(4):347-355.

171 Taghbalout, A., et al. Enhanced CRISPR-based DNA demethylation by Casilio-ME-mediated

172 RNA-guided coupling of methylcytosine oxidation and DNA repair pathways. Nature

173 communications 2019;10(1):4296.

174 